\title{
Thermal Energy Storage in a Confined Aquifer: Experimental Results
}

\author{
F. J. Molz, A. D. Parr, P. F. ANdersen, ANd V. D. Lucido \\ Civil Engineering Department, Auburn University, Auburn, Alabama 36830
}

J. C. WARMAN

Water Resources Research Institute, Auburn University, Auburn, Alabama 36830

\begin{abstract}
To aid in testing the idea of storing thermal energy in aquifers, an experiment was performed by Auburn University in which $54,784 \mathrm{~m}^{3}$ of water was pumped from a shallow supply aquifer, heated to an average temperature of $55^{\circ} \mathrm{C}$, and injected into a deeper confined aquifer where the ambient temperature was $20^{\circ} \mathrm{C}$. After a storage period of 51 days, $55,345 \mathrm{~m}^{3}$ of water were produced from the confined aquifer. Throughout the experiment, which lasted approximately 6 months, groundwater temperatures were recorded at six depths in each of 10 observation wells, and hydraulic heads were recorded in five observation wells. In order to prevent errors due to thermal convection, most of the observation wells recording temperature had to be backfilled with sand. During the 41-day production period, the temperature of the produced water varied from $55^{\circ}$ to $33^{\circ} \mathrm{C}$, and $65 \%$ of the injected thermal energy was recovered. At no time was an appreciable amount of free thermal convection observed in the storage formation. The dominant heat dissipation mechanisms appeared to be hydrodynamic thermal dispersion and possible mixing of cold and hot water induced by clogging and unclogging of the injection-production well. On the basis of laboratory and field studies, it was concluded that clogging of the injection well, which constituted the major technical problem during the experiment, was caused by the freshwater-sensitive nature of the storage aquifer. Due to the relatively low concentration of cations in the supply water, clay particles would swell, disperse, and migrate until they became trapped in the relatively small pores connecting the larger pores. Surging the pump and back washing the injection well would dislodge the clogging particles and temporarily improve the storage formation permeability. The phenomenon seems largely independent of temperature because it was reproduced in the laboratory with unheated water. It may, however, depend on pore velocity. Future research should be directed toward procedures for selecting storage aquifers that will have minimal susceptibility to clogging and other geochemical problems. Procedures for overcoming such difficulties are needed also because clogging and related phenomena will be more the rule than the exception. Designing an aquifer thermal storage system for maximum energy recovery would involve selecting an appropriate aquifer, analyzing the effects of hydrodynamic thermal dispersion and thermal convection if it is predicted to occur, anticipating geochemical problems, designing the optimum supply-injection-production well configuration and injecting a sufficiently large volume of heated water to realize economies of scale related to increasing volume-surface area ratio.
\end{abstract}

\section{INTRODUCTION}

The possibility of using aquifers as natural storage devices for thermal energy in the form of heated water has been considered seriously for the past 6 years [Meyer and Todd, 1973; Hausz and Meyer, 1975; Meyer, 1976; Meyer et al., 1976; Lawrence Berkeley Laboratory, 1978]. Related problems involving the natural drift of fluids from the storage zone have been considered also [Molz and Bell, 1977; Whitehead and Langhetee, 1978]. Several experimental studies have been performed with the objective of field testing the aquifer storage concept. Mathey [1978] conducted a 5-month injection-production cycle involving an unconfined aquifer with a natural temperature of $11^{\circ} \mathrm{C}$. Injection of $494 \mathrm{~m}^{3}$ of water at $51^{\circ} \mathrm{C}$ over a 9.3-day period was followed by four months of storage and a 28-day recovery of $16,370 \mathrm{~m}^{3}$. Extensive mixing of the hot and cold water, aided by thermal convection in the aquifer, led to a recovery of approximately $40 \%$ of the thermal energy injected. A second small experiment involving an unconfined aquifer was performed by Werner and Kley [1977]. Injection of $430 \mathrm{~m}^{3}$ of water took place over a 64-day period. Temperatures were monitored carefully, and a computer model was fitted to the data. No recovery pumping was attempted.

Results of a larger experiment involving a confined aquifer

Copyright (c) 1979 by the American Geophysical Uniou.

Paper number 9W0913.

0043-1397/79/009W-0913\$01.00 between depths of 40 and $62 \mathrm{~m}$ were reported by Molz et al. [1978]. The objective of this experiment was to begin testing the feasibility of thermal energy storage in confined aquifers and to provide data for the calibration of mathematical models describing the simultaneous transport of water and heat in the saturated zone [Papadopulos and Larson, 1978]. In preparation for the experiment, data related to subsurface geometry, hydraulic, and thermal properties were collected, and a well field, consisting of one injection-production well and 14 observation wells, was constructed near Mobile, Alabama. The subsequent injection of $7570 \mathrm{~m}^{3}$ of $37^{\circ} \mathrm{C}$ filtered water from an electric power plant thermal discharge canal resulted in a $67 \%$ thermal energy recovery after a storage period of 36.6 days. The injection process was plagued by serious clogging due to the inability to remove clay and small silt-sized particles by filtration alone. Ultimately, the injection was terminated when increased injection pressure, induced by the clogging, led to failure of the upper confining layer along a well casing.

The objective of the present communication is to describe the results of a second experiment at the Mobile test site which used essentially the same well field facilities which were constructed prior to the preliminary experiment [Molz et al., 1978]. The only modifications involved reinstrumentation of the observation wells, pressure grouting of the original injection well and the well where confining layer failure occurred, 


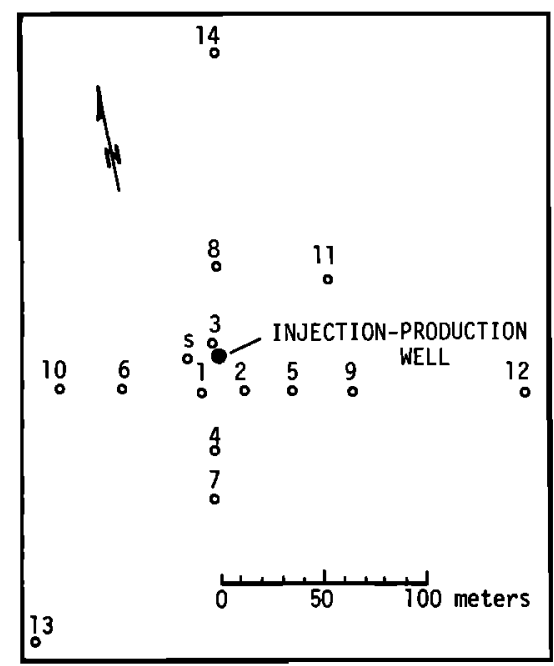

Fig. 1. Top view of the existing well field at the Mobile site drawn to scale.

construction of a new injection-production well, and construction of one additional observation well. In addition, supply water was obtained from an overlying, semiconfined aquifer and heated with an oil-fired boiler prior to injection.

\section{DESCRIPTION OF EXPERIMENT}

The project site is located in a soil borrow area at the Barry Steam Plant of the Alabama Power Company, about $20 \mathrm{mi}$ north of Mobile, Alabama (see Molz et al. [1978] for details). Shown in Figure 1 is a top view of the well field. The injection-production well is indicated and wells 1-14 are observation wells. Temperatures and hydraulic heads were recorded in the inner array of 11 observation wells which are located within the thermal radius of influence. Wells 12,13 , and 14 were used to observe the hydraulic conditions at what is arbitrarily called the boundary of the system. The hydraulic data were recorded primarily for use in calibrating existing numerical models and will not be presented in detail herein.

The system for supplying, heating, and injecting water is shown schematically in Figure 2. Supply water is pumped from a sandy gravel aquifer located between 25 and $34 \mathrm{~m}$ below the surface. The water is then metered and passed through an oil-fired boiler which raises its temperature from

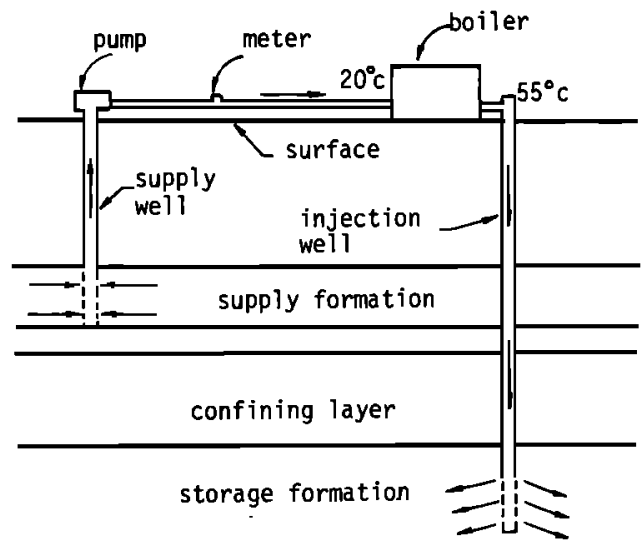

Fig. 2. Diagram of the system for supplying, heating, and injecting water into the storage aquifer.

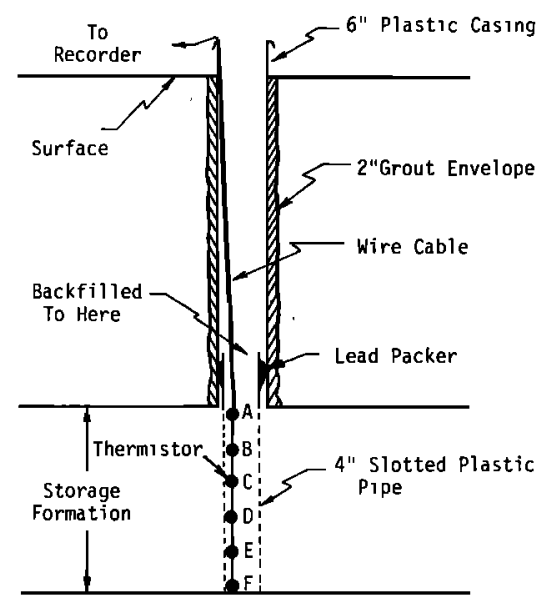

Fig. 3. Side view of a typical observation well showing details of the well construction and the position of the six thermistors used to record groundwater temperatures.

approximately $20^{\circ}$ to $55^{\circ} \mathrm{C}$. Injection into the storage formation, which extends from 39.6 to $61 \mathrm{~m}$ below the land surface, takes place through a 9.14-m long stainless steel screen. The storage formation is composed of a medium sand containing approximately $15 \%$ silt and clay by weight. Aquitards above and below are composed of several different types of clays.

With the exception of well 10 , each of the inner observation wells was equipped with thermistors to measure groundwater temperatures at six depths. Shown in Figure 3 is a diagram of a typical observation well. Plastic materials were used for well construction, and the thermistors were spaced uniformly in the vertical dimension of the storage aquifer.

The experiment itself involved a 6-month injection-storagerecovery cycle. Shown in Figure 4 is the cumulative injection volume as a function of time. Injection began on March 18 , 1978 , and continued until June 5, 1978, a total of 79 days. An injection rate varying from $12.61 \mathrm{~s}^{-1}(200 \mathrm{gpm})$ to $6.31 \mathrm{~s}^{-1}$ (100 gpm) resulted in an injection volume of $54,800 \mathrm{~m}^{3}$ at an average temperature of $55^{\circ} \mathrm{C}$ as shown in Figure 5. A 50.5-day storage period began on June 5, 1978, and was terminated on July 26 when production began and continued for 41 additional days. As shown in Figure 6, the pumping rate was nearly constant at $15.81 \mathrm{~s}^{-1}$ (250 gpm), except for uncontrolled power failures, and resulted in a production volume of 55,300 $\mathrm{m}^{3}$. Pumping was stopped when the production temperature reached $33^{\circ} \mathrm{C}$, which was $13^{\circ} \mathrm{C}$ above the ambient groundwater temperature.

\section{RESULTS OF EXPERIMENT}

Shortly after injection began, it became evident that thermal convection was occurring in the observation well closest

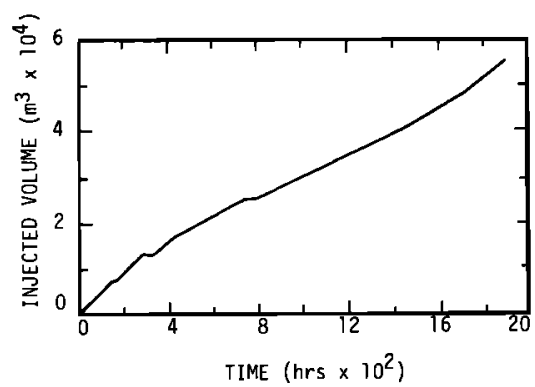

Fig. 4. Plot of cumulative injection volume as a function of time. The horizontal segments are due to pump shut-downs resulting from power failures and other problems. 


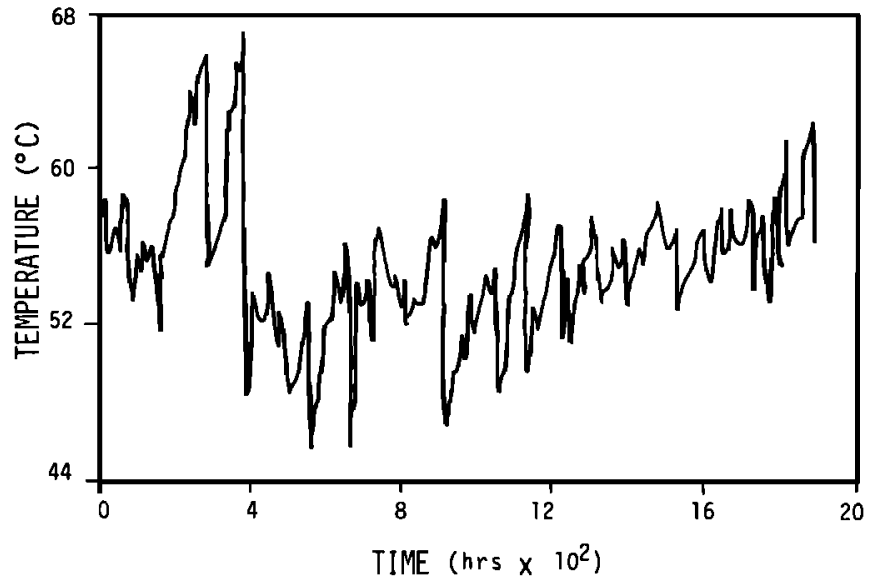

Fig. 5. Plot of injection temperature as a function of time.

to the injection well. When injection was stopped, the temperature in the observation well fell about $15^{\circ} \mathrm{C}$ in a few hours. Upon resuming injection, the temperature returned to its original value as fresh formation water was pushed into the well. Evidently, the temperature recorded by the thermistors was the result of a dynamic equilibrium between formation water moving horizontally through the well screen and thermal convection occurring in the well bore. Convection was controlled by backfilling each observation well within the thermal radius of influence with a clean medium sand. After this was done, the thermistors stabilized and recorded more representative nonuniform temperature distributions in the vertical dimension.

Shown in Figure 7 are typical vertical temperature profiles recorded in well 5 as a function of time. There is no evidence of thermal convection in the storage formation, and the temperature distribution remained relatively static throughout the storage period. This differs from the results of Mathey [1977], who observed significant convection in his experiment. The differing results are understandable if one considers the Rayleigh numbers for the two porous media, which largely determine when thermal convection will become appreciable [Wooding, 1957, 1962; Katto and Masuoka, 1967; Nield, 1968]. As the Rayleigh number increases, free thermal convection becomes more likely. Since this number for a porous system is proportional to the hydraulic conductivity, the Rayleigh number in the vertical dimension for Mathey's sandy gravel would be considerably' larger than the corresponding number for our medium sand-silt-clay material. Thus for roughly the same temperature regime, appreciable thermal convection could occur in one medium and not in the other.

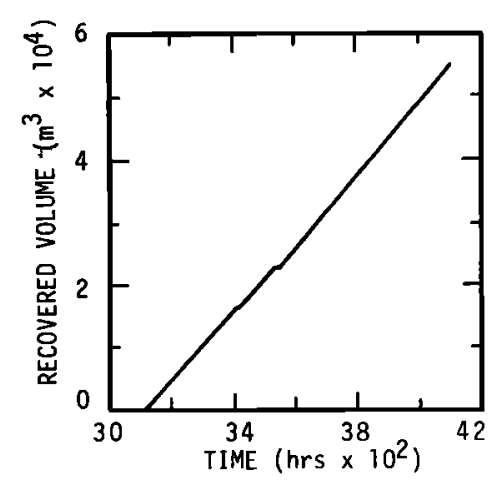

Fig. 6. Cumulative volume of water produced from the injection/ production well as a function of time.

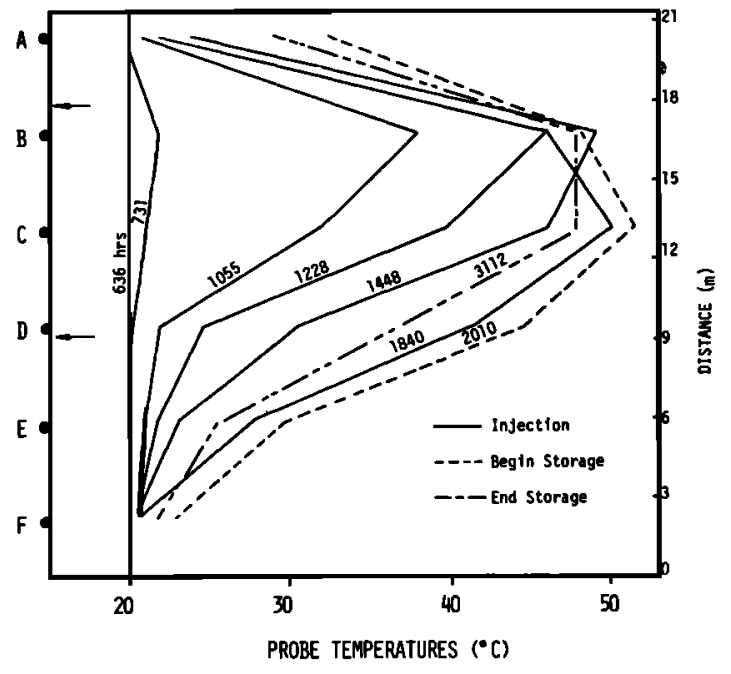

Fig. 7. Plots of vertical temperature distributions recorded in well 5. The number next to the curves denotes the time in hours after the start of injection, and the arrows indicate the elevation of the screen in the injection/production well. Letters A-F indicate thermistor positions.

As indicated in Figure 2, the screen of the injection well only partially penetrates the storage formation. Its vertical position corresponds to the elevations indicated by the arrows in Figure 7. This and the observation that the horizontal permeability appears to be an order of magnitude larger than the vertical permeability [Papadopulos and Larson, 1978] explain the relatively sharp peak in the Figure 7 temperature distributions. Presumably, the temperature plots would be more nearly uniform with depth if the injection well were fully penetrating.

Shown in Figures 8 and 9 are temperature distributions in the vertical plane containing observation wells $1,3,4,7$, and 8. Given the fact that the injection well is not in the center of the observation well field (Figure 1), this plane is as close as possible to a vertical cross-sectional view containing the injection well. The distribution at the end of the injection period shown in Figure 8 is distorted slightly to the left. This is opposite to the direction of natural groundwater drift and is most likely due to a high permeability zone in the aquifer matrix. By the end of the storage period, the distribution shifted to the right as shown in Figure 9. This is nearly the direction of regional groundwater flow. Measurements made before injection was started indicated a natural pore velocity from left to right in Figures 8 and 9 of approximately $0.05 \mathrm{~m} \mathrm{day}^{-1}$. Such a velocity would result in a total liquid drift of $2.3 \mathrm{~m}$ during

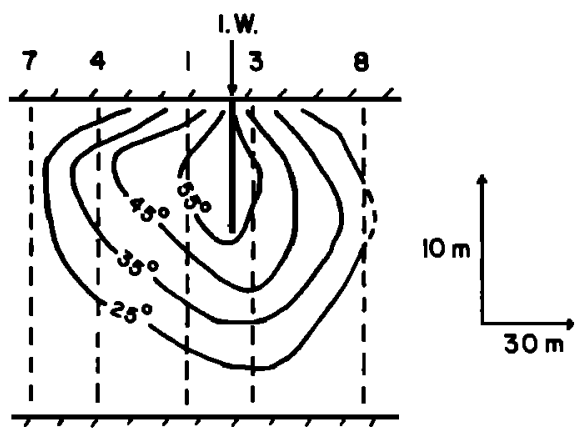

Fig. 8. Temperature distribution near the end of the injection period in a vertical plane containing wells $7,4,3$ and 8 . The vertical scale is exaggerated as indicated in the figure. 


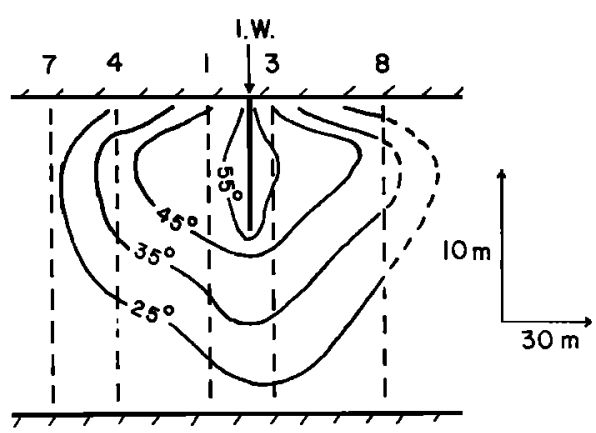

Fig. 9. Temperature distribution near the end of the storage period in a vertical plane containing wells $7,4,3$, and 8 . The vertical scale is exaggerated as indicated in the figure.

the 46.5-day time span between the distributions shown in Figures 8 and 9. Drift of the temperature contours should be less than this in proportion to the ratio of the liquid and matrix heat capacities. This, coupled with thermal dispersion, is the probable explanation for the slight shift in the temperature distribution recorded in the figures.

Shown in Figure 10 is the temperature of the water pumped from the storage aquifer as a function of time. The relatively rapid decrease of temperature with time is probably due mainly to heat conduction and mixing between the warm injected water and the cold native groundwater (hydrodynamic thermal dispersion). An effect of this magnitude would not be unexpected given the nonhomogeneous nature of the storage aquifer and the partially penetrating injection well (Figure 1). However, as discussed later in this communication, clogging of the injection-production well was observed during injection. If the clogging was nonuniform, certain portions of the formation might be partially prevented from receiving hot water. Upon starting production, the clogging might be reversed rather quickly, and relatively cold water could be pulled into the production well through the newly opened areas. This effect was observed by Brown and Silvey [1977] in their study of freshwater storage in a saline aquifer.

From the recorded temperature and the flow rate of the injected water as functions of time, one can calculate the amount of thermal energy injected above the ambient temperature. A similar calculation can be made for the amount of energy recovered. The mathematical expressions are given by

$$
\begin{aligned}
& E_{\mathrm{in}}=\int_{t_{\mathrm{u}}}^{t_{1}} \rho C\left[T_{i}(t)-T_{a}\right] I(t) d t \\
& E_{\mathrm{out}}=\int_{t_{2}}^{t_{3}} \rho C\left[T_{r}(t)-T_{a}\right] R(t) d t
\end{aligned}
$$

where $\rho$ is water density, $C$ is specific heat of water, $T_{,}(t)$ is temperature of injection water, $I(t)$ is injection rate, $T_{r}(t)$ is temperature of recovered water, $T_{a}$ is ambient groundwater temperature, and $R(t)$ is recovery rate. The limits $t_{0}, t_{1}$ and $t_{2}$, $t_{3}$ correspond to the time periods of injection and recovery, respectively, and, in general, $\rho$ and $C$ are functions of temperature. In practice, the exact expressions given by (1) are replaced by the finite difference approximations:

$$
\begin{aligned}
& E_{\mathrm{in}}=\frac{\bar{\rho} \bar{C}}{2} \sum_{n}\left[T_{i}\left(t_{n}\right)+T_{i}\left(t_{n+1}\right)-2 T_{a}\right]\left(Q I_{n+1}-Q I_{n}\right) \\
& E_{\mathrm{out}}=\frac{\bar{\rho} \bar{C}}{2} \sum_{m}\left[T_{r}\left(t_{m}\right)+T_{r}\left(t_{m+1}\right)-2 T_{a}\right]\left(Q R_{m+1}-Q R_{m}\right)
\end{aligned}
$$

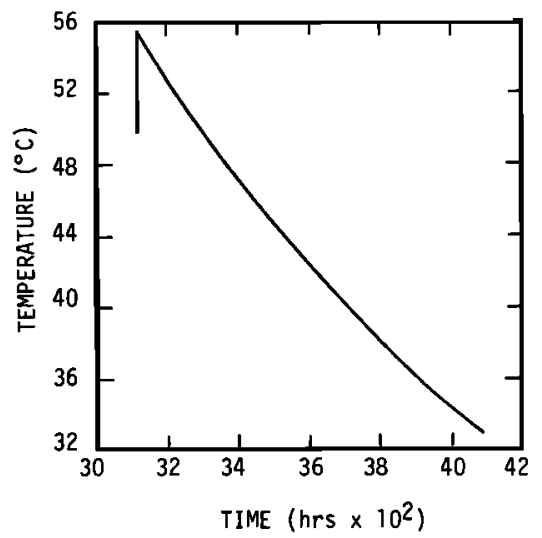

Fig. 10. Temperature of the water pumped from the storage formation as a function of time.

where $Q I$ is volume of water injected as a function of time, $Q R$ is volume of water produced as a function of time, and the 'bar' notation indicates average values of $\rho$ and $C$. Each value of the indices $n$ or $m$ corresponds to a time when temperature and cumulative injection or recovery data were recorded.

Shown in Figure 11 is a plot of the thermal energy recovery factor as a function of recovery temperature. This factor is defined as the ratio of $E_{\text {out }}$ at any time during production to $E_{\mathrm{nn}}$. When production was first started, the water temperature was $55^{\circ} \mathrm{C}$ and, of course, no energy had been recovered. By the time the water temperature dropped to $45^{\circ} \mathrm{C}, 31 \%$ of the injected energy had been recovered. Ultimately, when production ceased at a water temperature of $33^{\circ} \mathrm{C}, 65 \%$ of the injected energy had been recovered and $55,300 \mathrm{~m}^{3}$ of water had been pumped from the storage aquifer. This volume is nearly equal to the $54,800 \mathrm{~m}^{3}$ injection volume. The recovery of $65 \%$ of the injected energy in the upper $63 \%$ of the temperature range and contained in a volume of water essentially equal to the injection volume is much better than previous results [Mathey, 1977; Molz et al., 1978], although still less than the predicted performance potential of aquifer storage systems designed for maximum energy recovery [Lawrence Berkeley Laboratory, 1978]. However, the energy left behind in the formation will increase the recovery fraction during subsequent injection-storage-recovery cycles.

\section{Technical Problems}

The major problem encountered during the experiment was clogging of the injection-production well during injection.

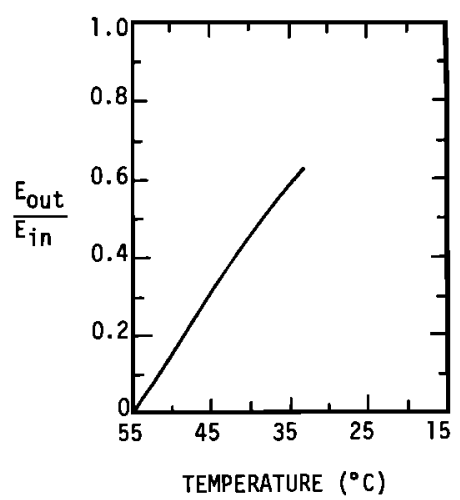

Fig. 11. Plot of the energy recovery factor, ratio of energy recovered at any time during production to total energy injected, as a function of production temperature. The plot shows that at the end of the production period, $65 \%$ of the injected thermal energy had been recovered. 


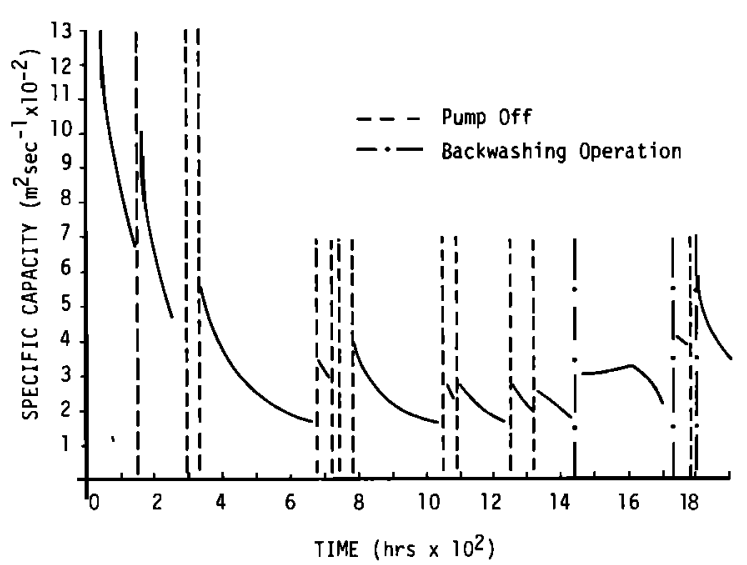

Fig. 12. Specific capacity of the injection well as a function of time. The specific capacity decreased markedly due to clogging of the formation around the injection well. Capacity was restored somewhat by turning the pump on and off and by backwashing the well.

Such a problem was observed in a preliminary experiment [Molz et al. 1978] but was at least partly of a different nature because of the relatively large suspended solids concentration in the supply water at that time. The present experiment utilized heated groundwater for injection which had a negligible concentration of suspended solids. Shown in Figure 12 is a plot of injection well specific capacity versus time. Because specific capacity is defined as the ratio of pumping rate to drawdown (or 'pushup' in the case of injection), this quantity would be expected to decrease with time. However, the magnitude and rate of decrease shown in Figure 12 are much too large to be completely explained in terms of the development of simple pushup in an aquifer of constant hydraulic conductivity. Pumping tests performed on wells equivalent to the present injection well, and the injection well itself during production, stabilized hydraulically within $\mathbf{4 8}$ hours after pumping began. This observation, along with a careful chemical analysis of the water in the supply and storage formation, which appears to eliminate corrosion or precipitation as a cause of specific capacity loss, indicates that the results shown in Figure 12 must be explained in terms of decreasing hydraulic conductivity of the aquifer matrix material. However, the spatial distribution of the conductivity loss is not known.

Further examination of Figure 12 shows that the specific capacity is sensitive to changes in injection rate. This was first observed during pump shut-downs due to power failures associated with thunderstorms. Later on, when well back-washing operations were initiated, even larger gains in specific capacity were realized. It was decided to determine if this type of flow rate-sensitive behavior could be duplicated in the laboratory using disturbed samples of storage aquifer material obtained during coring operations. Typical results obtained with a commercially available, constant-head permeameter are shown in Table 1 . The saturated hydraulic conductivity would normally decrease markedly with time. A brief surge in head gradient through the sample would increase the conductivity, sometimes by nearly an order of magnitude. Tapping the permeameter also had the effect of increasing the permeability for a short period of time. Results similar to those displayed in Table 1 were obtained using tap water.

It seems likely that the observed decrease in hydraulic conductivity is due to clay particle dispersion, migration, and subsequent blocking of the relatively small pores connecting larger pores. The temporary increase in conductivity caused by a sudden increase in head gradient would be due to the flushing out of a significant number of the clogging particles. Such an explanation is consistent with chemical analyses of water samples obtained from the supply aquifer and the storage aquifer. Most cations were more concentrated in water from the storage aquifer, although both waters were well undersaturated. Ions such as $\mathrm{Ca}, \mathrm{Fe}$, and $\mathrm{Mn}$ occurred in concentrations of less than $1 \mathrm{mg}^{-1}$ in both waters. $\mathrm{Si}$ and $\mathrm{Na}$ were in the $10 \mathrm{mg}^{-1}$ range in the storage formation water, which was 2 or 3 times more concentrated than in the supply water. A storage formation water alkalinity of $175 \mathrm{mg} \mathrm{I}^{-1}$ as $\mathrm{CaCO}_{3}$, versus about $6 \mathrm{mg} \mathrm{l}^{-1}$ as $\mathrm{CaCO}_{3}$ for the supply water, supports the conclusion that the ionic strength of the supply water is significantly less than that of the formation water. It is well known that clays in equilibrium with water having a high ionic concentration have a potential to swell and disperse when placed in contact with water having a relatively low concentration of ions. The phenomenon is initiated by osmotic swelling [van Olphen, 1963, p. 127] and has been observed many times in both the laboratory and the field [Brown and Silvey, 1977]. Aquifers which contain susceptible clays are called freshwater sensitive.

Additional evidence for clay swelling and dispersion was obtained during the production period. Soon after production pumping began, it was noticed that the recovered water contained a material that was causing a slight discoloration. Filtering of water samples along with chemical analyses and $X$ ray diffraction studies of the filter cake showed it to be composed of both kaolinite and smectite clays. These materials were definitely not being pumped into the storage formation from the supply aquifer. Therefore they must have originally been part of the storage aquifer matrix. Clay was produced from the storage formation during the entire production period, although the concentration decreased with time. It is estimated that a few thousand pounds of clay material was removed during production.

A second problem encountered while conducting the experiment was due to lightning discharges in the near vicinity of the site. Bolts of lightning induced voltages in the lines leading to the observation wells that were of sufficient magnitude to damage the recording equipment. This occurred even though the wires were shielded and the system grounded. On the average, the automatic recording system was down once

TABLE 1. Saturated Hydraulic Conductivity $(K)$ Values at Several Different Times Measured in a Constant-Head Permeameter Using a $10 \mathrm{meq}^{-1} \mathrm{CaCl}_{2} \cdot 2 \mathrm{H}_{2} \mathrm{O}$ Distilled Water Solution

\begin{tabular}{ccc}
\hline \multicolumn{1}{c}{ Date } & $K, \mathrm{~cm} / \mathrm{hr}$ & $T,{ }^{\circ} \mathrm{C}$ \\
\hline June 3, 1978 & 0.260 & 22 \\
June 5, 1978 & 0.206 & 24 \\
June 6, 1978 & 0.154 & 23 \\
June 7, 1978 & 0.118 & 24 \\
June 8, 1978 & 0.171 & 24 \\
June 9, 1978 & 0.120 & 24 \\
June 12, 1978 & 0.068 & 23 \\
June 13, 1978 & 0.059 & 23 \\
June 15, 1978 & 0.047 & 24 \\
June 20, 1978 & 0.078 & 26 \\
June 20, 1978 & $0.328^{*}$ & 25 \\
June 23, 1978 & 0.103 & 20 \\
June 23, 1978 & $0.291^{*}$ & 20 \\
\hline
\end{tabular}

Temperatures $(T)$ refer to the percolating solution which is in equilibrium with the laboratory temperature. A single sample was tested.

*A large hydraulic head difference was temporarily applied to increase the flow. 
every week. Consequently, a great deal of manual data tabulation was performed; the problem itself was never really solved.

\section{SUMMARY AND CONCLUSIONS}

Injection of water into a confined aquifer began on March 18, 1978, and continued until June 5, 1978. Prior to injection, the water was heated with an oil-fired boiler to an average temperature of $55^{\circ} \mathrm{C}$. Ultimately, $54,784 \mathrm{~m}^{3}$ of water were pumped into the storage aquifer. Clogging of the formation surrounding the injection well reduced the injection rate from the planned $200 \mathrm{gal} \mathrm{min}^{-1}\left(7571 \mathrm{~min}^{-1}\right)$ to as low as $100 \mathrm{gal}$ $\min ^{-1}\left(3781 \mathrm{~min}^{-1}\right)$. Injection was $95 \%$ continuous, however, with short stops due to power failures, a burned-out transformer, and three short backwashings (Figure 12). During the injection period, and throughout the entire experiment, groundwater temperatures were recorded at six depths in each of 10 observation wells, and hydraulic heads were recorded in five observation wells. In order to prevent significant thermal convection, most of the observation wells recording temperatures had to be backfilled with sand.

After a 51-day storage period, during which time there was only a small amount of regional groundwater drift, pumping of water from the storage aquifer began at a nearly constant rate of $250 \mathrm{gal} \mathrm{min}^{-1}\left(9461 \mathrm{~min}^{-1}\right)$. Initially, the temperature of the recovered water was $55^{\circ} \mathrm{C}$ and diminished at an average rate of $0.5^{\circ}$ per day. Very little heat was lost from the storage formation through the confining layers. Hydrodynamic thermal dispersion within the storage aquifer was probably an important dissipation process. Additional mixing due to clogging and unclogging of the formation could have played an important role also. On September 5, 1978, recovery ceased after a total of $55,345 \mathrm{~m}^{3}$ of water and $65 \%$ of the injected thermal energy above ambient had been recovered.

On the basis of laboratory and field studies, it was concluded that the clogging mentioned previously, which caused a large decrease in the specific capacity of the injection well, was due to the freshwater-sensitive nature of the storage aquifer. Clay particles would swell, disperse, and migrate until they became trapped in the relatively small pores connecting the larger pores. Thus the clogging phenomenon is viewed as a colloid chemistry problem largely independent of temperature. It may, however, depend on pore velocity. Except for waters high in calcium, moderate increases in temperature would be expected to increase the hydraulic conductivity rather than decrease it. Of course, other detrimental reactions such as those related to corrosion and scaling would tend to increase with temperature.

Future research should be directed toward establishing methods for controlling clogging problems in storage aquifers. This problem area is of prime importance, and injection difficulties are likely to be the rule rather than the exception in future aquifer storage systems. Several possibilities for minimizing clogging include increasing the ionic concentration of the injection water and using the storage formation itself as a source of supply water [Molz et al., 1978]. Research should also be directed toward procedures for selecting storage aquifers that will have minimum susceptibility to clogging and other geochemical problems.

The appropriate time may have arrived for subjecting the aquifer thermal storage concept to a fully elaborated experimental test. Using available theoretical and experimental information, a thermal energy storage system could be designed to maximize the energy recovery factor. This would involve selecting an ideal aquifer, analyzing the effects of hydrodynamic thermal dispersion and thermal convection if it is predicted to occur, anticipating geochemical problems, designing the optimum supply-injection-production well configuration, and injecting a sufficiently large volume of heated water to realize economies of scale related to increasing volume-surface area ratio.

At the present time, a second 6-month injection-storage-recovery cycle is being performed at the Mobile site. Further data are being collected, and the expected increase in the energy recovery factor with multiple storage cycles will be measured. Computer simulations of the first injection-storage-recovery cycle are being performed at the Lawrence-Berkeley Laboratory and should contribute to a more detailed future analysis of the data.

Acknowledgments. This work was made possible through the financial support of the U.S. Department of Energy (contract 7338). The Alabama Power Company allowed us to conduct the experiment on their land and also maintained the roads leading to the site. This help is gratefully acknowledged. In addition, the writers would like to thank Rod Jenkins, Associate Professor of Civil Engineering, for making the chemical analyses reported in this paper and Jacob Dane, Assistant Professor of Agronomy and Soils, for performing laboratory permeability measurements.

\section{REFERENCES}

Brown, D. L., and W. D. Silvey, Artificial recharge to a freshwatersensitive brackish-water sand aquifer, Norfolk, Virginia, U.S. Geol. Surv. Prof. Pap. 939, 1977.

Hausz, W., and C. F. Meyer, Energy conservation: Is the heat storage well the key? Public Utilities Fortnightly, 95, 34-38, 1975.

Katto, Y., and T. Masuoka, Criterion for the onset of convective flow in a fluid in a porous medium, Int. J. Heat Mass Transfer, 10, 297$309,1967$.

Lawrence Berkeley Laboratory, Thermal energy storage in aquifers workshop, LBL Rep. 8431, Berkeley, Calif., 1978.

Mathey, B., Development and resorption of a thermal disturbance in a phreatic aquifer with natural convection, J. Hydrol., 34, 315-333, 1977.

Meyer, C. F., Status report on heat storage wells, Water Resour. Bull., 12, 237-252, 1976.

Meyer, C. F., and D. K. Todd, Heat-storage wells, Water Well J., 27, 35-41, 1973.

Meyer, C. F., W. Hausz, B. L. Ayres, and H. M. Ingram, Role of the heat storage well in future U.S. energy systems, Rep. GE76TMP-27, General Elec. Tempo, Center for Advan. Stud., Santa Barbara, Calif., 1976.

Molz, F. J., and L. C. Bell, Head gradient control in aquifers used for fluid storage, Water Resour. Res., 13, 795-798, 1977.

Molz, F. J., J. C. Warman, and T. E. Jones, Aquifer storage of heated water, 1, A field experiment, Ground Water, 16, 234-241, 1978.

Nield, D. A., Onset of thermohaline convection in a porous medium, Water Resour. Res., 4, 553-560, 1968.

Papadopulos, S. S., and S. P. Larson, Aquifer storage of heated water, 2, Numerical simulation of field results, Ground Water, 16, 242-248, 1978.

van Olphen, H., An Introduction to Clay Colloid Chemistry, John Wiley, New York, 1963.

Werner, D., and W. Kley, Problems of heat storage in aquifers, J. Hydrol., 34, 35-43, 1977.

Whitehead, W. R., and E. J. Langhetee, Use of bounding wells to counteract the effects of pre-existing groundwater movement, $\mathrm{Wa}$ ter Resour. Res., 14, 273-280, 1978.

Wooding, R. A., Steady state free thermal convection of liquid in a saturated porous medium, J. Fluid Mech., 2, 273-285, 1957.

Wooding, R. A., Free convection of fluid in a vertical tube filled with porous material, J. Fluid Mech., 13, 129-144, 1962.

(Received February 19, 1979;

revised June 1, 1979;

accepted June 18, 1979.) 